\title{
Theoretical study of influence of electromagnetic stirring on transport phenomena in wire feed laser beam welding
}

Cite as: J. Laser Appl. 32, 022026 (2020); https://doi.org/10.2351/7.0000069

Submitted: 01 April 2020 . Accepted: 01 April 2020 . Published Online: 29 April 2020

Xiangmeng Meng, Antoni Artinov, Marcel Bachmann (D), and Michael Rethmeier (D)

\section{COLLECTIONS}

Paper published as part of the special topic on Proceedings of the International Congress of Applications of Lasers \& Electro-Optics (ICALEO<sup>\&\#174; </sup> 2019)

Note: This paper is part of the Special Collection: Proceedings of the International Congress of Applications of Lasers

(B)

\& Electro-Optics (ICALEO 2019).
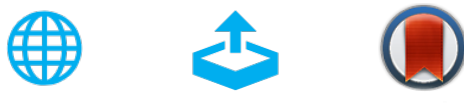

\section{ARTICLES YOU MAY BE INTERESTED IN}

Mathematical modeling of the geometrical differences between the weld end crater and the steady-state weld pool

Journal of Laser Applications 32, 022024 (2020); https://doi.org/10.2351/7.0000068

Influence of welding parameters on electromagnetic supported degassing of die-casted and wrought aluminum

Journal of Laser Applications 32, 022031 (2020); https://doi.org/10.2351/7.0000064

Effect of vacuum on penetration and defects in laser welding

Journal of Laser Applications 13, 187 (2001); https://doi.org/10.2351/1.1404413

\section{Seilight Highlights of the best new research} in the physical sciences 


\title{
Theoretical study of influence of electromagnetic stirring on transport phenomena in wire feed laser beam welding
}

Cite as: J. Laser Appl. 32, 022026 (2020); doi: 10.2351/7.0000069

Submitted: 1 April 2020 - Accepted: 1 April 2020 .

Published Online: 29 April 2020

Xiangmeng Meng, ${ }^{7}$ Antoni Artinov, ${ }^{7}$ Marcel Bachmann, ${ }^{7}$ (D) and Michael Rethmeier ${ }^{1,2}$ (D)

\author{
AFFILIATIONS \\ ${ }^{1}$ BAM Federal Institute for Materials Research and Testing, Unter den Eichen 87, Berlin 12205, Germany \\ ${ }^{2}$ Institute of Machine Tools and Factory Management, Technical University Berlin, Pascalstraße 8-9, Berlin 10587, Germany
}

Note: This paper is part of the Special Collection: Proceedings of the International Congress of Applications of Lasers \& Electro-Optics (ICALEO ${ }^{\circledR}$ 2019).

\begin{abstract}
The additional element from the filler wire in the laser beam welding is usually distributed inhomogeneously in the final weld due to the high solidification rate of weld pool. It has been found that the electromagnetic stirring produced by an external oscillating magnetic field can enhance the material mixing in the weld pool to achieve a more uniform element distribution. However, the magnetic field has a highly nonlinear and multicoupled interaction with the weld pool behavior, which makes the quantitative explanation of the physical mechanism difficult. In this study, the effect of electromagnetic stirring on the transport phenomena in the wire feed laser beam welding is investigated by a numerical modeling. A 3D transient multiphysical model considering the magnetohydrodynamics, heat transfer, fluid flow, keyhole dynamics, and element transport is developed. The multiple reflections and the Fresnel absorption of the laser on the keyhole wall are calculated using the ray tracing method. The numerical results show that a Lorentz force produced by the oscillating magnetic field and its induced eddy current gives significant influence on the transport phenomena in the molten pool. The forward and downward flow is enhanced by the electromagnetic stirring, which homogenizes the distribution of the additional elements from a nickel-based filler wire in a steel weld pool. The numerical results show a good agreement with the high-speed images of the molten pool, the fusion line from the optical micrograph, and the element distribution from the energy dispersive $\mathrm{x}$-ray spectroscopy. This work provides a physical base for the electromagnetic-controlled laser beam welding and some guidance for the selection of electromagnetic parameters.
\end{abstract}

Key words: magnetohydrodynamics, molten pool dynamics, element transport, laser beam welding

Published under license by Laser Institute of America. https://doi.org/10.2351/7.0000069

\section{INTRODUCTION}

When the laser power density exceeds a critical value (typically more than $1 \mathrm{MW} / \mathrm{cm}^{2}$ ), a steep crater named as keyhole will form under the recoil pressure of the highly evaporated metal during laser beam welding (LBW). Compared with typical arc welding, the LBW has several attractive advantages including deeper penetration, lower heat input, higher welding speed, and correspondingly, narrower heat affected zone and finer grain size. ${ }^{1}$ By using the additional filler metal in LBW, mostly in the form of wire, further improvements can be achieved, such as good adaptability for the gap tolerance and proper metallurgical control. ${ }^{2}$ However, it has been found that the added material may not be transferred to the bottom of the molten pool due to the exceedingly cooling rate during LBW, especially for the thick plate of more than $5 \mathrm{~mm} .{ }^{3}$ It results in the deterioration of the homogeneity of the weld property.

The electromagnetic control which is widely used in the material processing provides a potential way to overcome this problem. The external magnetic field was first applied to the LBW by Kern et al. It was found that the humping defect occurring at high welding speed was supressed. ${ }^{4}$ When a transverse oscillating magnetic field was implemented at the backside of the workpiece, an effective support against the gravity was produced to prevent the root sagging in the full penetration LBW. ${ }^{5}$ The study of Fritzsche et al. suggested that the 
oscillating magnetic field can also produce an electromagnetic Archimedes force on the bubbles in the molten pool. The bubble's floating was enhanced, and the porosity was reduced dramatically in the partial penetration LBW. ${ }^{6}$ Gatzen employed a coaxial magnetic field to the wire feed LBW (WFLBW) of Al alloy. The additional Si from the filler wire was well mixed in the molten pool under the induced electromagnetic stirring (EMS).

Although the beneficial effect of the magnetic field on the LBW has been identified experimentally, the involved physical mechanism is still not clear due to the absence of the quantitative temperature and velocity data of the molten pool. It can be ascribed to two aspects: first, the molten pool is covered by bright metal vapor during LBW and the liquid metal is nontransparent, which makes the experimental measurement difficult and second, the flowing liquid metal in the molten pool has highly multicoupled and nonlinear interactions with the magnetic field.

Recently, the researchers have made some multiphysical modeling efforts to investigate the quantitative relationship between molten pool behavior and the magnetic field in LBW. Bachmann et al. built a 3D steady-state multiphysical model of LBW with the magnetic field. The effects of electromagnetic support and electromagnetic braking on the molten pool behavior in the LBW of both ferromagnetic and nonferromagnetic materials were analyzed. ${ }^{8,9}$ Chen et al. studied the effect of thermoelectric current in an electromagnetically supported LBW by a steady-state numerical model. It was indicated that the Lorentz force induced by the thermoelectric current and magnetic field had a non-negligible influence on the fluid flow in the molten pool. ${ }^{10}$ Gatzen et al. investigated the transport phenomena in the WFLBW with a coaxial magnetic field numerically. The enhancement of material mixing in the molten pool due to electromagnetic stirring was explained. ${ }^{11}$ However, the surface of the molten pool was not deformable, and the keyhole was fixed as a cylindrical or conical shape in all the aforementioned models. The important impact of free surface deformation and keyhole evolution was not considered.

In this paper, a $3 \mathrm{D}$ transient numerical heat transfer and fluid flow model coupled with free surface tracking, magnetic induction, and element transport is developed for WFLBW with electromagnetic stirring (). A self-consistent ray tracing method is used to calculate the multiple reflections and the Fresnel absorption of the laser on the keyhole wall. The model is well verified by experimental results. The influence of electromagnetic stirring on the transport phenomena in the molten pool is analyzed and discussed in detail.

\section{EXPERIMENTAL PROCEDURE}

The AISI 304 stainless steel that had an austenite microstructure was chosen as a base metal. A nickel-based alloy, Inconel 625, was used as a filler wire. The base metal was $200 \mathrm{~mm}$ in length, $60 \mathrm{~mm}$ in width, and $10 \mathrm{~mm}$ in thickness. The filler wire had a diameter of $1.2 \mathrm{~mm}$. A significant difference of $\mathrm{Ni}$ content exists between the base metal and the filler wire ( $8.7 \%$ vs. $58.0 \%)$, so the $\mathrm{Ni}$ can be used as a marking element to describe the material mixing in the molten pool.

Figure 1(a) gives the schematic of the welding system. The weld is generated by an IPG YLR 20000 fiber laser system. Butt
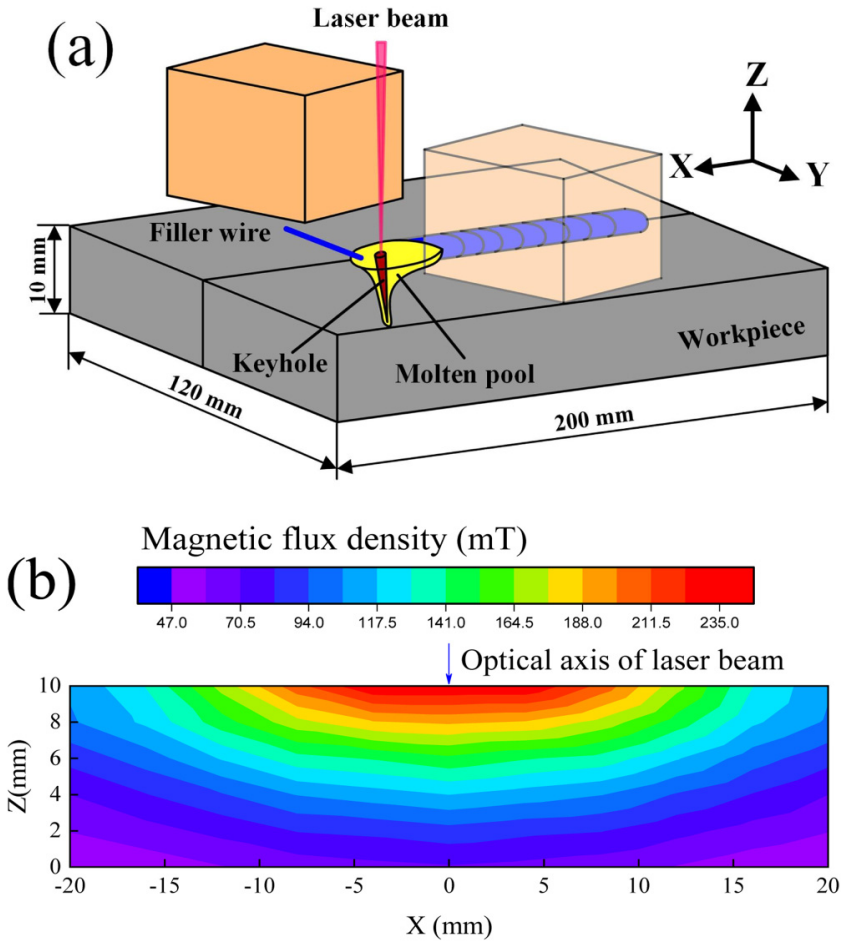

FIG. 1. EMS-WFLBW system: (a) schematic of EMS-WFLBW and (b) magnetic flux density.

configuration was carried out without any gap. The wire was fed to the leading edge of keyhole with $57^{\circ}$ angle with respect to the optical axis of laser. Argon 5.0 in purity of $99.999 \%$ was provided behind the laser spot. The welding parameters used are listed in Table I. An in-house alternating current electromagnet was fixed at the position of $2 \mathrm{~mm}$ above the base metal and had a $75^{\circ}$ angle with respect to the welding direction. The electromagnet produced a $3600 \mathrm{~Hz}$ oscillating magnetic field to induce effective electromagnetic stirring in the molten pool. The cross section of the core has a dimension of $16 \times 16 \mathrm{~mm}^{2}$, and the distance between two poles is $20 \mathrm{~mm}$. The magnetic flux density was measured in the cold metal testing. A small gap existed between the two workpieces, and the

TABLE I. Welding parameters used in experiment and simulation.

\begin{tabular}{lc}
\hline \hline Laser power & $7.5 \mathrm{~kW}$ \\
Laser spot radius at focal plane & $0.26 \mathrm{~mm}$ \\
Wavelength & $1070 \mathrm{~nm}$ \\
Focal length & $350 \mathrm{~mm}$ \\
Focal position & $-3 \mathrm{~mm}$ \\
Rayleigh length & $5.3 \mathrm{~mm}$ \\
Welding speed & $1.3 \mathrm{~m} / \mathrm{min}$ \\
Wire feeding speed & $2.1 \mathrm{~m} / \mathrm{min}$ \\
Shielding gas & $201 / \mathrm{min}$ \\
\hline
\end{tabular}


magnetic field was measured by a Hall sensor along the longitudinal section.

The observation specimens were cut from the weld specimens after the experiment. They were grinded and polished mechanically first. Then, a V2A solution that contained $10 \mathrm{ml}$ hydrochloric acid, $10 \mathrm{ml}$ hydrogen nitrate, and $100 \mathrm{ml}$ water was used to etch the prepared surface at a temperature of $50{ }^{\circ} \mathrm{C}$ for $30 \mathrm{~s}$. The optical microscopy observation was performed on the transverse section to reveal the fusion zone shape. Energy-dispersive x-ray spectroscopy (EDX) mapping was conducted to measure the $\mathrm{Ni}$ content on the transverse section.

\section{MATHEMATICAL MODELLING}

\section{A. Magnetohydrodynamic theory}

The magnetohydrodynamics theory suggests that a current density will be induced if a magnetic field is applied to a flowing electrically conducting fluid, e.g., liquid metal in the molten pool, which gives ${ }^{12}$

$$
\vec{j}=\sigma_{\mathrm{e}}(\vec{E}+\vec{v} \times \vec{B}),
$$

where $\sigma_{\mathrm{e}}$ is the electrical conductivity, $\vec{E}$ is the induced electric field, $\vec{v}$ is the flow velocity, and $\vec{B}$ is the magnetic flux density. The external magnetic field and its induced current produce a volumetric Lorentz force,

$$
\vec{F}_{L}=\vec{j} \times \vec{B}
$$

The Lorentz force has two components: one from the temporal variation of the magnetic field, $\vec{E}$ term in Eq. (1), and one from electromagnetic braking, $\vec{v} \times \vec{B}$ term in Eq. (1). Therefore, by applying a suitable magnetic field, different effects such as stirring, supporting, or deceleration can be produced in the molten pool.

\section{B. Governing equations}

The physical phenomena involved in the EMS-WFLBW are extremely complex. Several assumptions are made without losing the key physical features. They include

(i) The fluid is assumed to be laminar and Newtonian. The density is constant, and buoyance is treated by Boussinesq approximation.

(ii) The relative magnetic permeability of the base metal (the 304 austenite steel) remains as one. The gaseous phase in the model is nonconductive.

(iii) The Joule heat and the Seebeck effect are not considered. The liquid metal transfer is not influenced by the Lorentz force. ${ }^{13}$

The governing equations of the multiphysical model in a fixed Cartesian coordinate can be written as below

- Mass conservation equation,

$$
\nabla \cdot \vec{v}=\frac{m_{\mathrm{w}}}{\rho}
$$

where $\rho$ is the density and $m_{\mathrm{w}}$ is the mass source of filler wire.
- Navier-Stokes equation,

$$
\rho\left(\frac{\partial \vec{v}}{\partial t}+\vec{v} \cdot \nabla \vec{v}\right)=-\nabla p+\mu \nabla^{2} \vec{v}-\mu K \vec{v}+m_{\mathrm{w}} \vec{v}_{\mathrm{w}}+\vec{S}_{\mathrm{m}}
$$

where $p$ is the pressure, $t$ is the time, $\mu$ is the dynamic viscosity, $\vec{v}_{\mathrm{w}}$ is the velocity of molten filler metal, and $K$ is the deceleration coefficient in the semisolid zone. Other momentum sources are implemented into $\vec{S}_{\mathrm{m}}$ term.

- Energy equation,

$$
\rho\left[\frac{\partial h}{\partial t}+(\vec{v} \cdot \nabla) h\right]=\nabla \cdot(k \nabla T)+h_{\mathrm{w}}+S_{q},
$$

where $h$ is the enthalpy, $T$ is the temperature, $k$ is the heat conductivity, and $h_{\mathrm{w}}$ is the energy source from the molten filler metal. Other energy sources are implemented into the $S_{q}$ term.

- Volume of fluid (VOF) equation,

$$
\frac{\partial F}{\partial t}+\nabla \cdot(\vec{v} F)=F_{\mathrm{w}}
$$

where $F$ is the volume fraction and $F_{\mathrm{w}}$ is the volume source from the filler wire.

- Magnetic induction equation,

$$
\frac{\partial \vec{b}}{\partial t}+(\vec{v} \cdot \nabla) \vec{b}=\frac{1}{\mu_{\mathrm{m}} \sigma_{\mathrm{e}}} \nabla^{2} \vec{b}+\left(\left(\vec{B}_{0}+\vec{b}\right) \cdot \nabla\right) \vec{v}-(\vec{v} \cdot \nabla) \vec{B}_{0}
$$

where $\mu_{\mathrm{m}}$ is the relative magnetic permeability, which is 1 in the current study, $\vec{B}_{0}$ is the external magnetic field produced by the AC electromagnet, and $\vec{b}$ is the secondarily induced magnetic field. The $\vec{B}_{0}$ is taken as an initial data input and moves with the laser spot during simulation.

$\S$ Scalar equation for Ni transport,

$$
\frac{\partial F_{\mathrm{s}} \rho_{\mathrm{s}} w_{\mathrm{Ni}}}{\partial t}+\nabla \cdot\left(F_{\mathrm{s}} \rho_{\mathrm{s}} \vec{v} w_{\mathrm{Ni}}-F_{\mathrm{s}} D_{\mathrm{Ni}} \nabla w_{\mathrm{Ni}}\right)=S_{\mathrm{Ni}}
$$

where $w_{\mathrm{Ni}}$ is the Ni weight percent, $F_{\mathrm{s}}$ is the volume fraction of the steel phase, $D_{\mathrm{Ni}}$ is the diffusion coefficient of $\mathrm{Ni}$ in iron, and $S_{\mathrm{Ni}}$ is the Ni source from the filler wire.

\section{EMS-WFLBW models}

In the WFLBW, part of the laser beam irradiates on the filler wire first. The filler metal will be melted and then transferred downward. Meanwhile, some energy is dissipated into the atmosphere due to the reflection on the melting front. The other part of the laser beam heats, melts, or even evaporates the base metal to form the molten pool and the keyhole.

According to the captured molten pool images, the transfer mode of liquid filler metal in this study is a continuous liquid 
bridge transfer. The average temperature of the liquid bridge $T_{\mathrm{w}}$ is assumed to be $2900 \mathrm{~K}^{14}$ The absorption ratio of the laser energy on the melting front of the filler wire $\eta_{\mathrm{w}}$ is determined as

$$
\eta_{\mathrm{w}}=\frac{\pi v_{\text {feed }} \rho_{\mathrm{w}} r_{\mathrm{w}}^{2}\left[c_{\mathrm{pw}}\left(T_{\mathrm{w}}-T_{0}\right)+\Delta H_{\mathrm{w}}\right]}{P_{\mathrm{L}}} \approx 7.2 \%,
$$

where $P_{\mathrm{L}}$ is the laser power, $v_{\text {feed }}$ is the wire feeding speed, $\rho_{\mathrm{w}}, c_{\mathrm{pw}}$, and $\Delta H_{\mathrm{w}}$ are the density, specific heat, and melting latent heat of Inconel 625 alloy, and $r_{\mathrm{w}}$ is the filler wire radius.

The dissipation ratio at the melting front of the filler wire $\eta_{\text {wloss }}$ can be calculated by the following regressive function: ${ }^{15}$

$$
\eta_{\text {wloss }}=2.07 P_{\mathrm{L}}^{-2.11} L_{\mathrm{F}}^{0.82} v_{\text {feed }}^{0.76} \approx 5.5 \% \text {, }
$$

where $L_{\mathrm{F}}$ is the focal length.

It is assumed that the rest of the laser energy still have a Gaussian-like axisymmetric distribution. At the focal plane, it gives

$$
q_{\mathrm{L}}(x, y)=\frac{2\left(1-\eta_{\mathrm{w}}-\eta_{\mathrm{wloss}}\right) P_{\mathrm{L}}}{\pi r_{\mathrm{f} 0}^{2}} \exp \left(-2 \frac{x^{2}+y^{2}}{r_{\mathrm{f} 0}^{2}}\right),
$$

where $r_{\mathrm{f} 0}$ is the laser beam radius at the focal plane.

A self-consistent ray tracing method proposed by Cho et al. is used in the study to calculate the multiple reflection and Fresnel absorption on the keyhole wall. ${ }^{16}$ The laser beam is discretized into numerous subrays first, and each subray has its own locationdependent energy density, as shown in Fig. 2(a). The initial path of the subray is defined by considering the diffraction of the laser beam. The laser beam radius along the optical axis can be calculated as

$$
r_{\mathrm{f}}(z)=r_{\mathrm{f} 0}\left[1+\left(\frac{z-z_{0}}{z_{\mathrm{r}}}\right)^{2}\right]^{1 / 2}
$$

where $z_{0}$ is the position of focal plane and $z_{\mathrm{r}}$ is the Rayleigh length.

The $n$th reflection direction $\vec{R}_{n}$ which is also the $n+1$ th incident direction $\vec{I}_{n+1}$ is determined by the $n$th incident direction $\vec{I}_{n}$ and the normal at the $n$th reflection point $\vec{N}_{n}$ on the transient keyhole surface, as shown in Fig. 2(b),

$$
\vec{R}_{n}=\vec{I}_{n+1}=\vec{I}_{n}-2\left(\vec{I}_{n} \cdot \vec{N}_{n}\right) \vec{N}_{n}
$$

The Fresnel reflection model is adopted to calculate the absorption rate as given below ${ }^{17}$

$$
\begin{aligned}
\alpha= & 1-\frac{1}{2}\left(\frac{1+\left(1-\varepsilon \cos \theta_{n}\right)^{2}}{1+\left(1+\varepsilon \cos \theta_{n}\right)^{2}}+\right. \\
& \left.\times \frac{\varepsilon^{2}-2 \varepsilon \cos \theta_{n}+2 \cos ^{2} \theta_{n}}{\varepsilon^{2}+2 \varepsilon \cos \theta_{n}+2 \cos ^{2} \theta_{n}}\right),
\end{aligned}
$$

where $\varepsilon$ is a material dependent coefficient and $\theta_{\mathrm{n}}$ is the incident angle at the nth incident point.

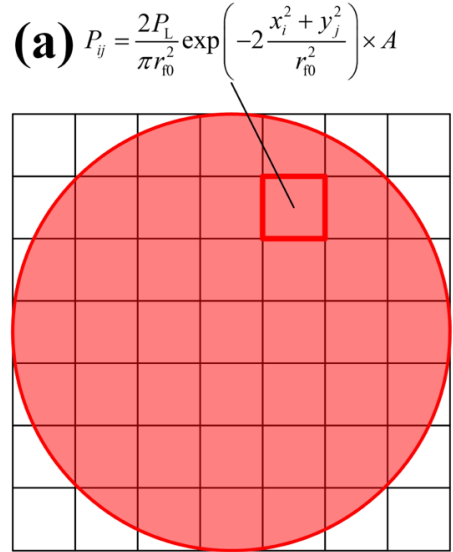

(b)

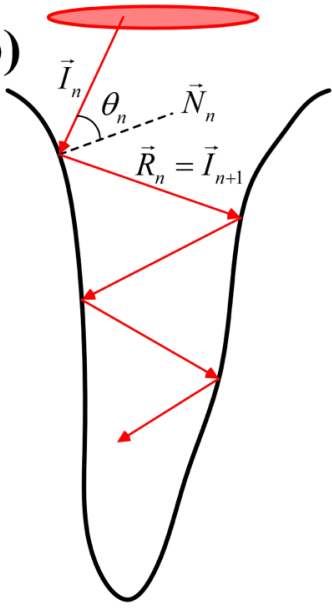

FIG. 2. Schematic of the ray tracing method: (a) discretization of laser beam energy and (b) multiple reflections on the keyhole wall.

The evaporation-induced recoil pressure, which is the dominant driving force for keyhole formation, can be calculated as ${ }^{18}$

$$
p_{r}=\frac{A B}{\sqrt{T}} \exp \left(-\frac{m_{a} \Delta L_{v}}{R T}\right)
$$

where $A$ is $3.9 \times 10^{12} \mathrm{~kg} / \mathrm{m} \cdot \mathrm{s}^{2}$ for pure iron and $B$ is $0.55 \mathrm{~K}^{1 / 2} . \Delta L_{\mathrm{v}}$ is the evaporation latent heat and $m_{\mathrm{a}}$ is the molar mass.

The surface tension-induced capillary pressure $p_{\mathrm{ca}}$ and thermo-capillary stress $\tau_{\mathrm{ma}}$ can be expressed as

$$
\begin{gathered}
p_{\mathrm{ca}}=\gamma \kappa, \\
\tau_{\mathrm{ma}}=\frac{\partial \gamma}{\partial T} \frac{\partial T}{\partial \vec{s}},
\end{gathered}
$$

where $\gamma$ is the surface tension coefficient, $\vec{s}$ is the tangential vector, and $\kappa$ is the curvature.

The volumetric forces are implemented as the momentum sources in Eq. (4), which gives

$$
\vec{S}_{\mathrm{m}}=\frac{1}{\mu_{m}} \nabla \times\left(\vec{B}_{0}+\vec{b}\right) \times\left(\vec{B}_{0}+\vec{b}\right)-\beta \rho\left(T-T_{0}\right) \vec{g}+\rho \vec{g}
$$

where $\vec{g}$ is the gravity acceleration vector, $\beta$ is the coefficient of thermal expansion, and $T_{0}$ is the room temperature. The terms at the right-hand side represents the Lorentz force, buoyance, and gravity, respectively.

The metal vapor with high temperature (up to $6000 \mathrm{~K}$ ) and high velocity (up to $200 \mathrm{~m} / \mathrm{s}$ ) also shows non-negligible influence on the keyhole dynamics. In this paper, the secondary heating effect of the vapor plume $q_{\text {plume }}$, the release of the evaporation latent heat due to the vapor's recondensation $q_{\text {recond }}$, the vapor-induced shear 
stress $\tau_{\text {vapor }}$, and stagnation pressure $p_{\text {vapor }}$ are considered empirically according to the works of Cho et al. ${ }^{16}$ and Muhammad et al. ${ }^{19}$

\section{Boundary conditions}

The energy balance between laser heat input, heat dissipation, and energy transferred into the workpiece can be written as

$$
\begin{aligned}
k \frac{\partial T}{\partial \vec{n}}= & q_{\mathrm{L}}-h_{\mathrm{c}}\left(T-T_{0}\right)-\sigma \varepsilon_{\mathrm{r}}\left(T^{4}-T_{0}^{4}\right)-\rho v_{\mathrm{evp}} \Delta L_{\mathrm{v}} \\
& +q_{\text {plume }}+q_{\text {recond }}
\end{aligned}
$$

where $q_{\mathrm{L}}$ is the spatial laser energy distribution, $h_{\mathrm{c}}$ is the heat convection coefficient, $\sigma$ is the Stefan-Boltzmann constant, and $\varepsilon_{\mathrm{r}}$ is the emissivity. $v_{\text {evp }}$ is the recession speed of evaporation ${ }^{20}$ and $\vec{n}$ is the normal vector.

The normal and tangential force balance on the keyhole can be expressed as follows

$$
\begin{aligned}
-p+2 \mu \frac{\partial v_{\mathrm{n}}}{\partial \vec{n}} & =-p_{\mathrm{r}}-p_{\text {vapor }}+p_{c a} \\
-\mu \frac{\partial v_{\mathrm{t}}}{\partial \vec{n}} & =\tau_{\mathrm{ma}}+\tau_{\text {vapor }}
\end{aligned}
$$

where $v_{\mathrm{n}}$ is the normal velocity and $v_{\mathrm{t}}$ is the tangential velocity.

The simulation domain is much smaller than the realistic workpiece in width and length. Thus, continuum boundary of heat conduction and electric conduction is applied on the side surface of the steel workpiece. ${ }^{21}$ The bottom of the steel workpiece is set as insulating.

\section{E. Numerical setup}

The simulated workpiece has dimensions of $30 \times 8 \times 10 \mathrm{~mm}^{3}$, and a gas phase layer of $2 \mathrm{~mm}$ thickness is set above it for the application of the VOF method. The central zone is equally divided into fine cells of $0.2 \mathrm{~mm}$. The outer zone has coarser cells, which gradually expands from 0.2 to $1.0 \mathrm{~mm}$.

The commercial software ANSYS FLUENT 18.2 based on the finite volume method is used to solve all the transport equations. The spatial discretization of momentum, energy, Ni content scalar, and magnetic field is realized by the second order upwind method, and the PressureImplicit with Splitting of Operators (PISO) algorithm is used for the coupling of velocity and pressure. The simulation program is run on a high-performance computing cluster, and $0.9 \mathrm{~s}$ real time welding process simulation takes about $60 \mathrm{~h}$ clock time calculation. The physical properties of the 304 stainless steel and the Inconel 625 are taken from the literature studies. ${ }^{22-25}$ The steel properties in the model are percentage-weighted averages of the base metal and the filler metal.

\section{RESULTS AND DISCUSSION}

\section{A. Electromagnetic behavior}

The 3D distribution of the current density is shown in Fig. 3. There are two circulating electric currents induced in the workpiece, which are almost separated by the high-temperature keyhole region.

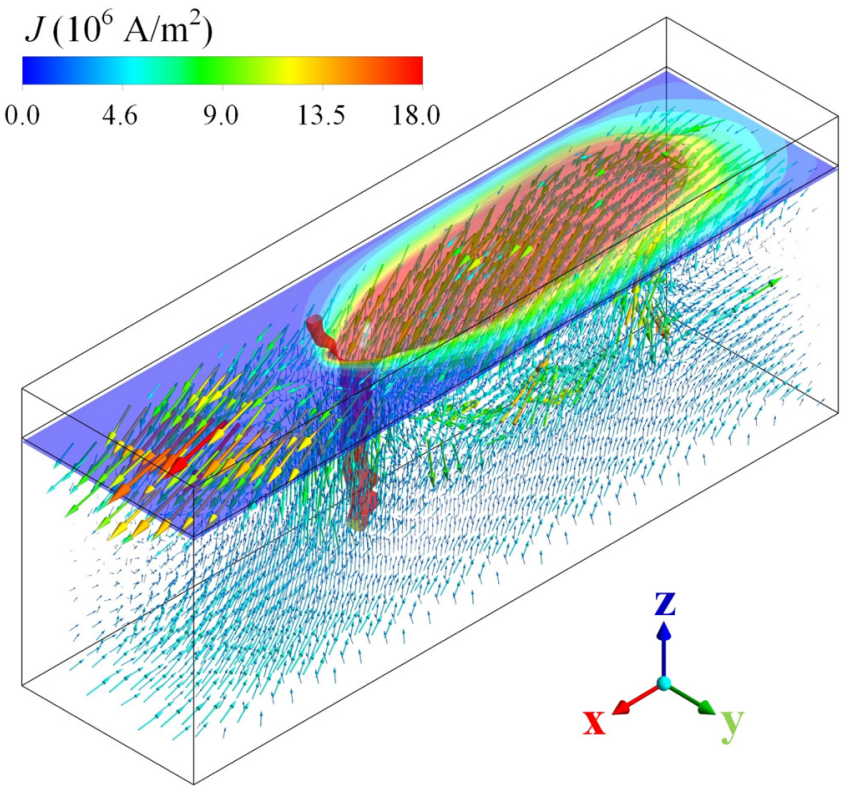

FIG. 3. Three-dimensional current density in the workpiece.

The current circulations have a $15^{\circ}$ with respect to the welding direction. The maximum current density occurs in the cold metal region in front of the keyhole due to the high conductivity there. The peak current density in the molten pool is about $1.4 \times 10^{7} \mathrm{~A} / \mathrm{m}^{2}$.

An oscillating Lorentz force is produced in the molten pool by the transient magnetic field and its induced eddy current, as shown in Fig. 4. At a certain time $\left(t=t_{0}\right)$, the maximum inward Lorentz force is formed with a peak value of $1.75 \times 10^{6} \mathrm{~N} / \mathrm{m}^{3}$. After onefourth of the magnetic field frequency $\left(6.9 \times 10^{-5} \mathrm{~s}\right)$, maximum outward Lorentz force occurs with a peak value of $1.44 \times 10^{6} \mathrm{~N} / \mathrm{m}^{3}$. The molten pool will be stirred by the Lorentz force to enhance the material mixing.

\section{B. Transport phenomena}

Figure 5 shows the drilling procedure of keyhole at the welding initial stage $(20-60 \mathrm{~ms})$. As the laser beam irradiates the workpiece surface, the base metal is heated, melted, and evaporated rapidly under the energy density of $\sim 10^{10} \mathrm{~W} / \mathrm{m}^{2}$. The molten pool surface is depressed under the downward recoil pressure to form a narrow and deep keyhole. Only a thin liquid layer exists at the front wall of keyhole, and the majority of the liquid metal is pushed backward to the rear part. The averaged drilling rate is about $100 \mathrm{~mm} / \mathrm{s}$, which agrees well with the available experimental results. ${ }^{26}$ At the initial stage, the volume of the molten pool is $<10 \mathrm{~mm}^{3}$, and the volumetric Lorentz force is easily masked by the strong recoil pressure. Hence, the electromagnetic stirring plays a minor role on the keyhole formation.

The calculated temperature distribution and velocity field at the top surface and the longitudinal section after reaching the quasistable state are given in Fig. 6. In WFLBW, the liquid filler metal 


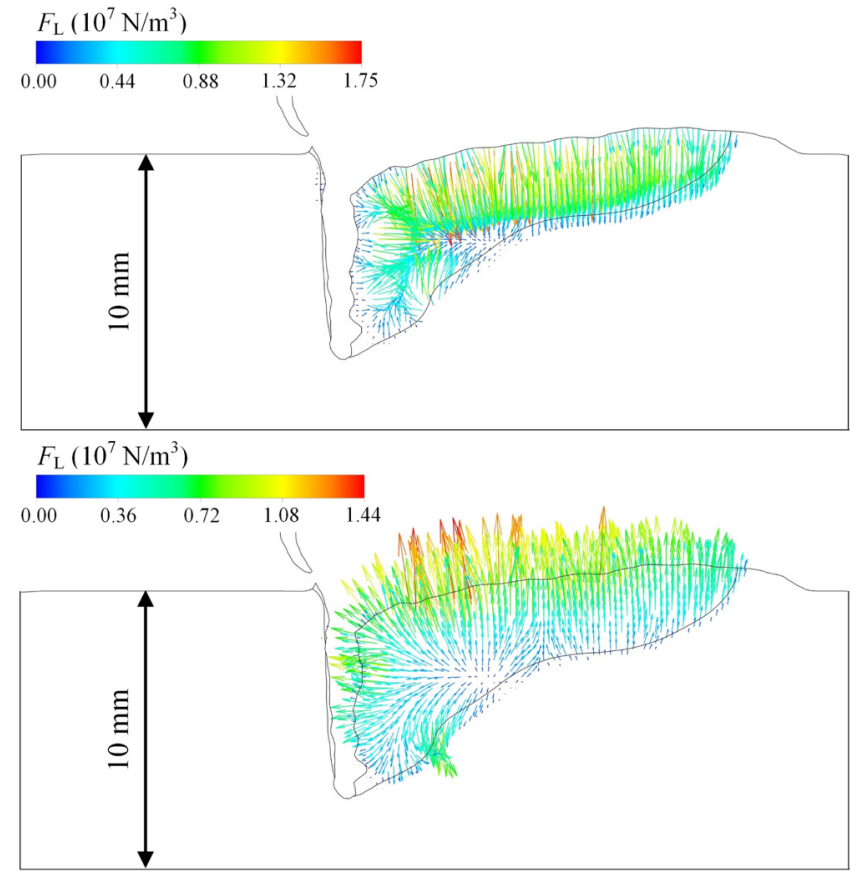

FIG. 4. Induced Lorentz force at the longitudinal section: (a) at $t_{0}$ and (b) $t_{0}+6.9 \times 10^{-5} \mathrm{~s}$.

with a velocity of $0.14 \mathrm{~m} / \mathrm{s}$ strikes the upside of the keyhole front wall. Then, the filler metal together with the melted base metal flow backward round the keyhole, and the average velocity is about $0.5 \mathrm{~m} / \mathrm{s}$. Therefore, the molten pool is significantly elongated at the upper part and has a typical tear-dropped shape. The flowing direction changes at the middle part of molten pool, and the liquid metal starts to flow forward along the longitudinal section, as shown in Fig. 6(a). This flow is identified as the main circulation in the weld pool.

It is found that the fundamental flowing pattern of the molten pool remains after introducing the electromagnetic stirring, by comparing Figs. 6(a) and 6(b). However, the backward flow is enhanced, and the length of main circulation increases from 7.7 to $10.2 \mathrm{~mm}$. More importantly, as the liquid metal flows toward the keyhole rear wall along the longitudinal section, its direction changes from forward to downward gradually. It can be expected that the downward flow is beneficial for the mixing of the filler metal in the molten pool. In addition, the velocity of the liquid metal is also increased by the electromagnetic stirring at the rear part of the molten pool.

The mixing of the filler metal in the final weld is determined directly by the thermal fluid flow in the molten pool. It can be characterized by the $\mathrm{Ni}$ distribution, considering the considerable difference of $\mathrm{Ni}$ content between the base metal and the filler wire. Figure 7(a) shows that most of the added filler metal concentrates at the upper part of the weld with a $3.1 \mathrm{~mm}$ depth in the WFLBW. It is because the liquid metal flow along the longitudinal section is (a)
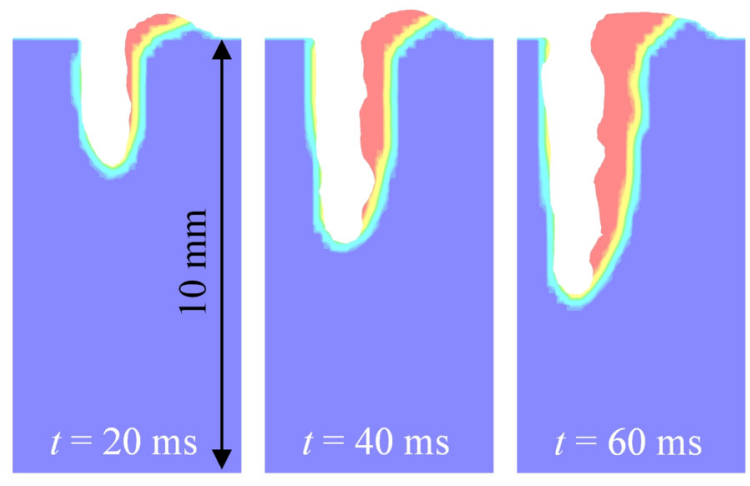

(b)

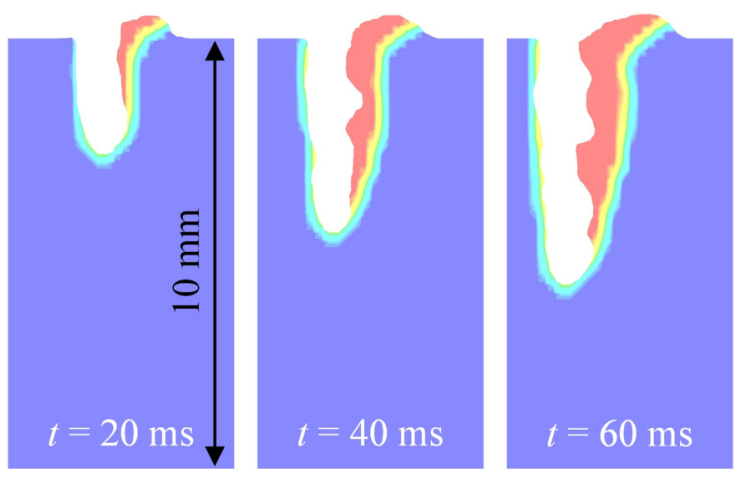

FIG. 5. Keyhole formation procedure: (a) WFLBW and (b) EMS-WFLBW (red: liquid meta, green: mushy zone, blue: solid metal).

almost parallel to the welding direction, and thereby, the filler metal cannot be transferred to the bottom of the molten pool. The $\mathrm{Ni}$ is well homogenized in the weld after the electromagnetic stirring is applied, as shown in Fig. 7(b). The depth of the well-mixed region increases to $5.9 \mathrm{~mm}$. The downward flow along the longitudinal section contributes to the enhancement of mixing.

The distribution of $\mathrm{Ni}$ at the longitudinal section is plotted in Fig. 8. As indicated in Fig. 6, the metal flow on the keyhole front wall is quite irregular rather than typically downward. Therefore, the keyhole front wall is not a crucial transfer channel although the liquid filler metal contacts it first. The $\mathrm{Ni}$ content is also not uniform along the welding direction in the WFLBW. Severe spiking phenomenon occurs at the lower part of weld, which may result from the keyhole instability. Under the stirring of the Lorentz force, this phenomenon is suppressed.

\section{Model verification}

The calculated contour line of welds and the experimentally measured ones are compared with Fig. 9 to verify the model's reasonability. The penetration depths are predicted accurately by the model for both processes. The calculated fusion zone and the reinforcement part of WFLBW are also in a considerably good agreement with the experimental result. A relatively larger error exists in 

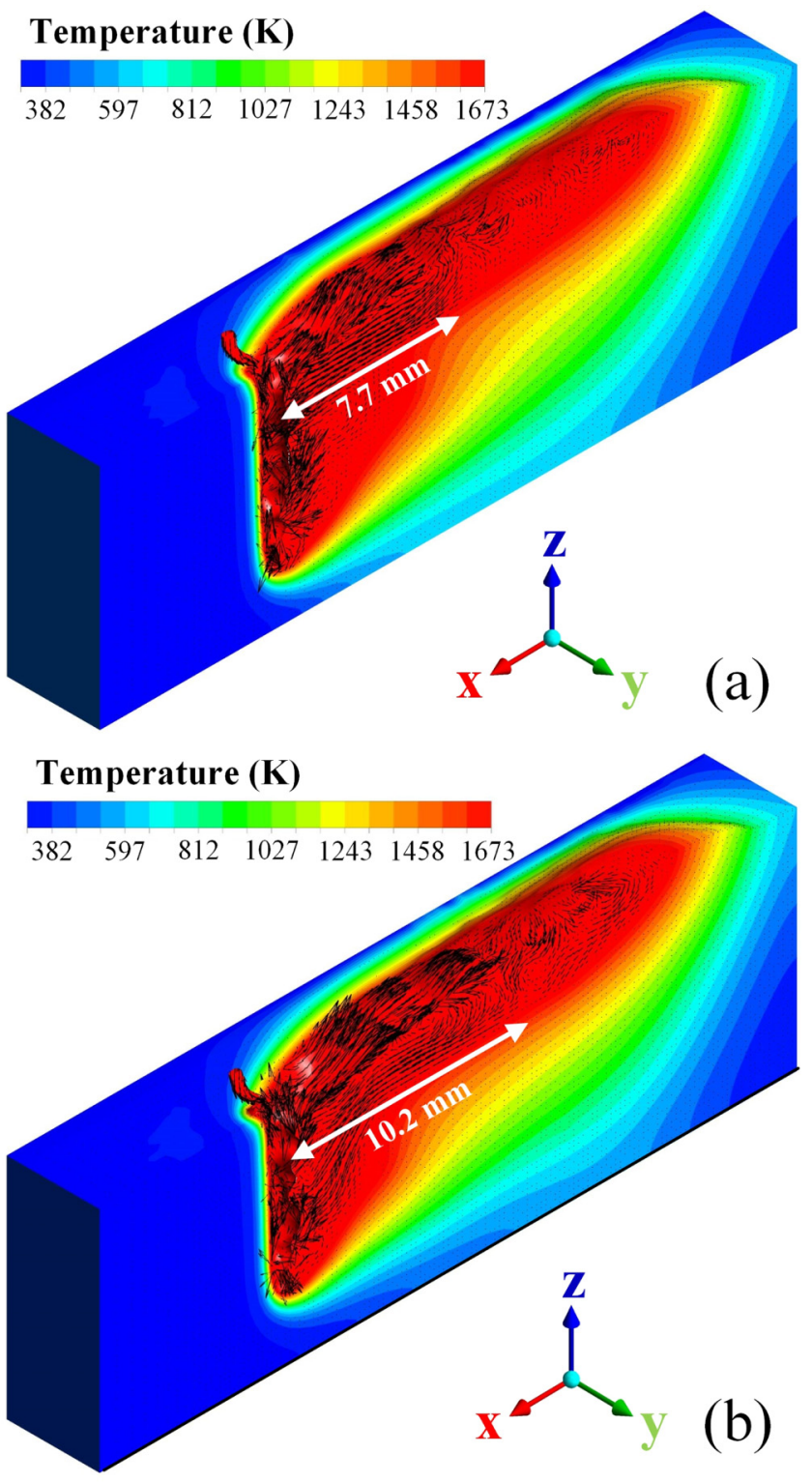

FIG. 6. Calculated three-dimensional temperature distribution and velocity field: (a) WFLBW and (b) EMS-WFLBW $(t=0.875 \mathrm{~s})$.

the upper fusion line of EMS-WFLBW, as shown in Fig. 9(b). The neglection of the influence of the Lorentz force on the filler metal transfer is inferred to cause the error.

Figure 10 gives the Ni distribution from the EDX mapping. It has been proved experimentally that the electromagnetic stirring enhances the mixing in the molten pool. There is a good agreement between the simulated and the measured results. The relative errors for the depth of well-mixed region are $7 \%$ and $15 \%$, respectively, by comparing Figs. 7 and 10.
Ni content (wt \%)

$\begin{array}{llllll}9.1 & 10.5 & 11.9 & 13.3 & 14.7 & 16.1\end{array}$

$\mathrm{Ni}$ content $(\mathrm{wt} \%)$

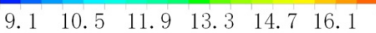
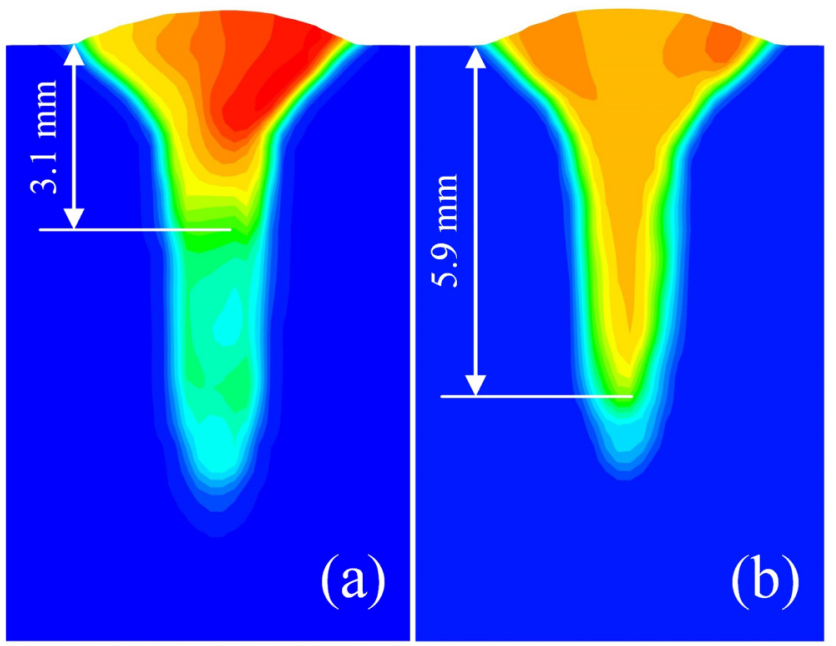

FIG. 7. Calculated distribution of Ni at the cross section: (a) WFLBW and (b) EMS-WFLBW.

\section{$\mathrm{Ni}$ content (wt \%)}

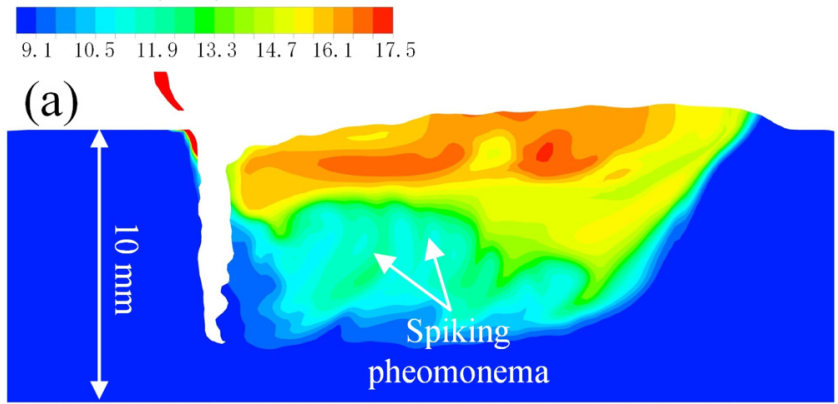

(b)

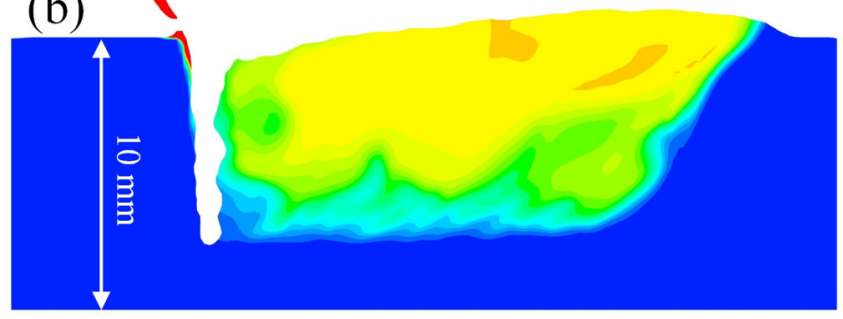

FIG. 8. Calculated distribution of Ni at the longitudinal section: (a) WFLBW and (b) EMS-WFLBW. 


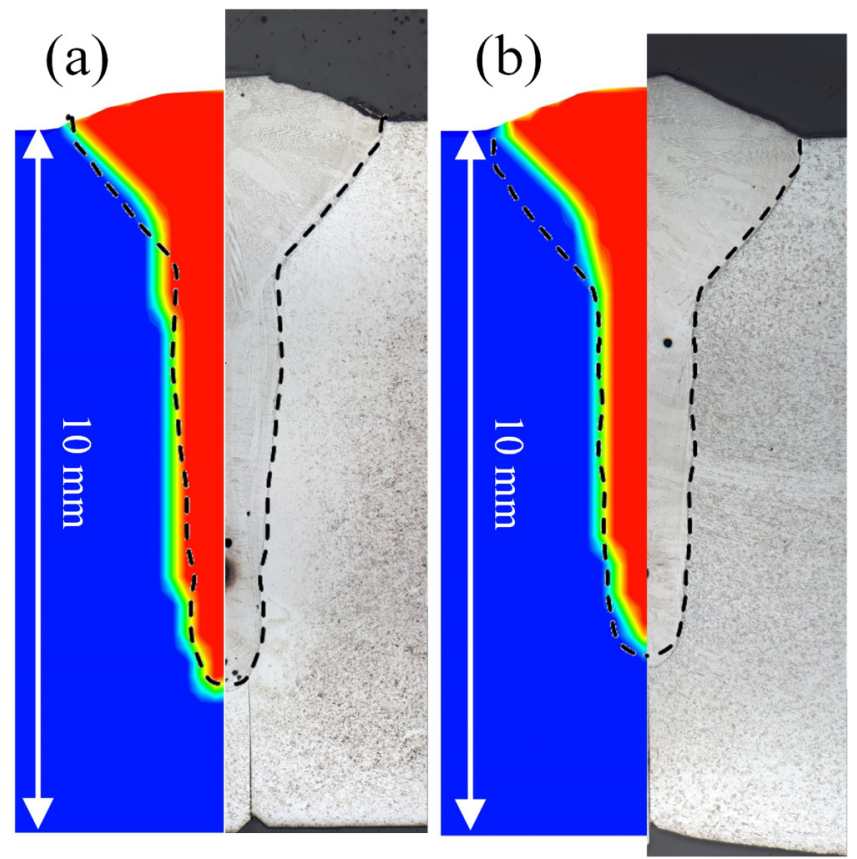

FIG. 9. Comparison between calculated and experimental fusion line shape: (a) WFLBW and (b) EMS-WFLBW.

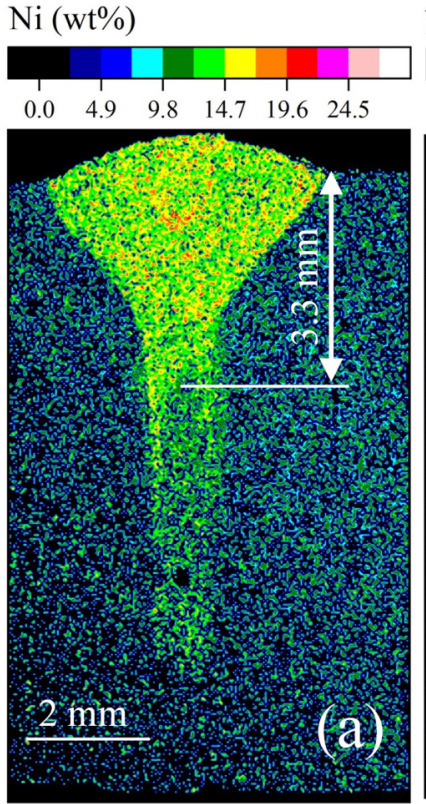

\section{$\mathrm{Ni}(w \mathrm{t} \%)$}
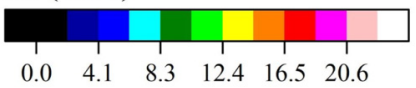

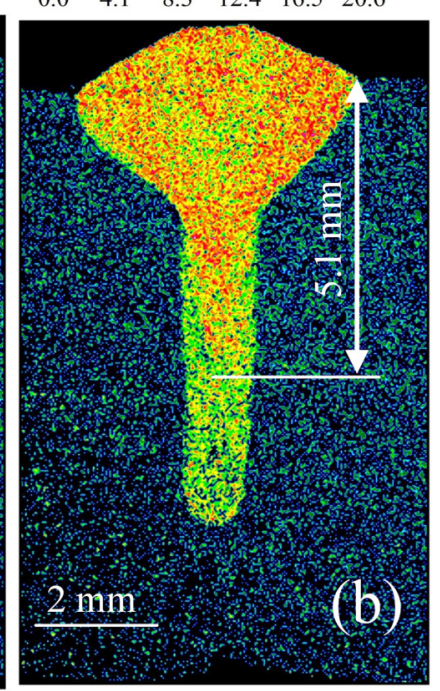

FIG. 10. Ni distribution from the EDX measurement: (a) WFLBW and (b) EMS-WFLBW.

\section{CONCLUSIONS}

(1) A multiphysical model is developed for the EMS-WFLBW to calculate the magnetic induction, heat transfer, fluid flow, element transport, and keyhole dynamics. A self-consistent ray tracing method is used to calculate the multiple reflections and Fresnel absorption on the keyhole wall.

(2) The oscillating magnetic field produces a periodic Lorentz force in the molten pool. It only shows a minor effect on the keyhole formation at the initial stage but influences the thermal fluid flow significantly after reaching the quasistable state.

(3) The filler material is well mixed in the final weld in EMS-WFLBW because the electromagnetic stirring enhances the downward flow along the longitudinal section.

\section{ACKNOWLEDGMENT}

This work is funded by the Deutsche Forschungsgemeinschaft (DFG, German Research Foundation)_Project No. 416014189.

\section{REFERENCES}

${ }^{1}$ M. Bachmann, A. Gumenyuk, and M. Rethmeier, "Welding with high-power lasers: Trends and developments," Phys. Procedia 83, 15-25 (2016).

${ }^{2}$ M. J. Torkamany, A. F. Kaplan, F. M. Ghaini, M. Vänskä, A. Salminen, K. Fahlström, and J. Hedegård, "Wire deposition by a laser-induced boiling front," Opt. Laser Technol. 69, 104-112 (2015).

${ }^{3}$ S. Gook, A. Gumenyuk, and M. Rethmeier, "Hybrid laser arc welding of X80 and X120 steel grade,” Sci. Technol. Weld. Join. 19, 15-24 (2014).

${ }^{4}$ M. Kern, P. Berger, and H. Huegel, "Magneto-fluid dynamic control of seam quality in $\mathrm{CO}_{2}$ laser beam welding," Weld. J. 79, 72-78 (2000).

${ }^{\mathbf{5}}$ V. Avilov, A. Fritzsche, M. Bachmann, A. Gumenyuk, and M. Rethmeier, "Full penetration laser beam welding of thick duplex steel plates with electromagnetic weld pool support," J. Laser Appl. 28, 022420 (2016).

${ }^{6}$ A. Fritzsche, K. Hilgenberg, F. Teichmann, H. Pries, K. Dilger, and M. Rethmeier, "Improved degassing in laser beam welding of aluminum die casting by an electromagnetic field," J. Mater. Process. Technol. 253, 51-56 (2018).

${ }^{7} \mathrm{M}$. Gatzen, "Influence of low-frequency magnetic fields during laser beam welding of aluminium with filler wire," Phys. Procedia 39, 59-66 (2012).

${ }^{8}$ M. Bachmann, V. Avilov, A. Gumenyuk, and M. Rethmeier, "About the influence of a steady magnetic field on weld pool dynamics in partial penetration high power laser beam welding of thick aluminium parts," Int. J. Heat Mass Transfer 60, 309-321 (2013).

${ }^{9}$ M. Bachmann, V. Avilov, A. Gumenyuk, and M. Rethmeier, "Experimental and numerical investigation of an electromagnetic weld pool support system for high power laser beam welding of austenitic stainless steel," J. Mater. Process. Technol. 214, 578-591 (2014).

${ }^{10}$ J. Chen, Y. Wei, X. Zhan, C. Gu, and X. Zhao, "Thermoelectric currents and thermoelectric-magnetic effects in full-penetration laser beam welding of aluminum alloy with magnetic field support," Int. J. Heat Mass Transfer 127, 332-344 (2018). ${ }^{11}$ M. Gatzen, Z. Tang, and F. Vollertsen, "Effect of electromagnetic stirring on the element distribution in laser beam welding of aluminium with filler wire," Phys. Procedia 12, 56-65 (2011).

${ }^{12} \mathrm{P}$. A. Davidson, An Introduction to Magnetohydrodynamics (Cambridge University Press, Cambridge, 2011), p. 2001.

${ }^{13}$ R. Hu, M. Luo, T. Liu, L. Liang, A. Huang, D. Trushnikov, and S. Pang, "Thermal fluid dynamics of liquid bridge transfer in laser wire deposition 3D printing," Sci. Technol. Weld. Joining 24, 401-411 (2019).

${ }^{14}$ Y. Arata, H. Maruo, I. Miyamoto, and R. Nishio, "High power $\mathrm{CO}_{2}$ laser welding of thick plate: Multipass weding with filler wire (welding physics, process \& instrument)," Trans. JWRI 15, 199-206 (1986). 
${ }^{15}$ A. S. Salminen and V. P. Kujanpää, "Effect of wire feed position on laser welding with filler wire," J. Laser Appl. 15, 2-10 (2003).

${ }^{16}$ W. I. Cho, S. J. Na, C. Thomy, and F. Vollertsen, "Numerical simulation of molten pool dynamics in high power disk laser welding," J. Mater. Process. Technol. 212, 262-275 (2012).

${ }^{17}$ W. Schulz, G. Simon, H. M. Urbassek, and I. Decker, "On laser fusion cutting of metals," J. Phys. D Appl. Phys. 20, 481-488 (1987).

${ }^{18} \mathrm{~V}$. Semak and A. Matsunawa, "The role of recoil pressure in energy balance during laser materials processing," J. Phys. D Appl. Phys. 30, 2541-2552 (1997).

${ }^{19}$ S. Muhammad, S. W. Han, S. J. Na, A. Gumenyuk, and M. Rethmeier, "Study on the role of recondensation flux in high power laser welding by computational fluid dynamics simulations," J. Laser Appl. 30, 012013 (2018).

${ }^{\mathbf{2 0}} \mathrm{H}$. Ki, J. Mazumder, and P. S. Mohanty, "Modeling of laser keyhole welding: Part I Mathematical modeling, numerical methodology, role of recoil pressure, multiple reflections, and free surface evolution," Metall. Mater. Trans. A 33, $1817-1830$ (2002).

${ }^{21}$ X. Meng, G. Qin, and R. Zong, "Thermal behavior and fluid flow during humping formation in high-speed full penetration gas tungsten arc welding," Int. J. Thermal Sci. 134, 380-391 (2018).

${ }^{22} \mathrm{Y}$. Su, Z. Li, and K. C. Mills, "Equation to estimate the surface tensions of stainless steels," J. Mater. Sci. 40, 2201-2205 (2005).

${ }^{23}$ D. Peckner and I. Bernstein, Handbook of Stainless Steels (McGraw-Hill Book Company, 1977), p. 1977.

${ }^{24} \mathrm{~K}$. C. Mills, Recommended values of Thermophysical Properties for Selected Commercial Alloys (Woodhead Publishing, 2002), p. 2002.

${ }^{25}$ K. D. Maglić, N. L. Perović, and A. M. Stanimirović, "Calorimetric and transport properties of Zircalloy 2, Zircalloy 4, and Inconel 625," Int. J. Thermophys. 15, 741-755 (1994).

${ }^{\mathbf{2 6}} \mathrm{S}$. Li, G. Chen, M. Zhang, Y. Zhou, and Y. Zhang, "Dynamic keyhole profile during high-power deep-penetration laser welding,” J. Mater. Process. Technol. 214, 565-570 (2014).

\section{Meet the Authors}

Xiangmeng Meng, born in 1990 in Dezhou, China, received his Ph.D. in the field of Material Science and Engineering from the
Shandong University at 2017. Currently, he is working as a postdoc researcher in the department "Welding Technology" in the BAM Federal Institute for Materials Research and Testing in Berlin, Germany. His research topics focus on the process development and numerical simulation of arc welding and laser beam welding.

Antoni Artinov, born in 1991 in Aytos (Bulgaria), received his M.Sc. in the field of Engineering Science from the Berlin Institute of Technology and his M.Sc. in Mechanical Engineering from the Tomsk Polytechnic University. Since 2016, he is working as a research assistant at the Federal Institute for Materials Research and Testing in Berlin, Germany in the department "Welding Technology." Currently, he is working in the field of numerical simulations in laser beam welding processes.

Dr.-Ing. Marcel Bachmann, born 1984 in Berlin, is with the BAM Federal Institute for Materials Research and Testing in Berlin, Germany in the department "Welding Technology" since 2009, where he is leading the working group "Welding Simulation." He received his diploma from the Berlin Institute of Technology in Engineering Science and his Ph.D. for numerical investigations of electromagnetically assisted high power laser beam welding processes. Currently, he is working on several projects involving numerical simulations in welding processes.

Prof. Dr.-Ing. Michael Rethmeier is with the BAM Federal Institute for Materials Research and Testing. He is the head of the division "Welding Technology." He is also heading the "Chair of Joining Technology" at the Institute of Machine Tools and Factory Management, Berlin Institute of Technology and is division director of "Joining and Coating Technology" at the Fraunhofer Institute for Production Systems and Design Technology. Present research topics among others include innovative arc welding processes, high power laser beam welding, and numerical simulations in various welding processes. 